\title{
Performance Enhancement of High Power High Repetition Rate Semiconductor Opening Switches
}

\author{
A.H. Abbasi, K. Niayesh* And J. Shakeri \\ School of Electrical and Computer Engineering, University of Tehran, Iran
}

\begin{abstract}
High power semiconductor opening switches (SOSs) with $\mathrm{P}^{+} \mathrm{PNN}^{+}$doping profile have been used in different pulsed power applications because of their fast high current interruption capability. In high pulsed power applications with high repetition rate, temperature increase of the semiconductor switch during its operation is no more negligible. In this paper, the cut-off characteristics of SOSs with different doping profiles are compared and then the impact of the temperature increase on the performance of the semiconductor opening switch is investigated using a detailed physical model. The simulation results indicate that due to the temperature increase of the switch, both the current amplitude and the cut-off characteristics of the switch are affected. Semiconductor opening switches with different base materials ( $\mathrm{Si}$ and $\mathrm{SiC}$ ) are studied. Due to favorable intrinsic characteristics of $\mathrm{SiC}$ (higher thermal conductivity, higher saturation velocity and higher breakdown electric field), the performance of the SOS can be enhanced using $\mathrm{SiC}$ as the base material and the design of compact high pulsed power applications can be achieved.
\end{abstract}

PACS numbers: 85.30.De

\section{Introduction}

One of the main challenges in using inductive energy storage to generate high pulsed power is to design a switch capable of fast interruption of high currents and holding off high recovery voltages. Recently, due to high repetition rate and reliability, Semiconductor Opening Switches with a doping profile of $\mathrm{P}^{+} \mathrm{PNN}^{+}$ have been used in different pulsed power applications. In the present paper the impact of different doping profiles on the performance of SOS is investigated and then the influence of temperature increase due to high repetition rate on the performance of the switch is demonstrated. Eventually semiconductor opening switches with different base materials ( $\mathrm{Si}$ and $\mathrm{SiC}$ ) are studied.

\section{Impact of varying diffusion length on the SOS performance}

To investigate the influence of different doping profiles on the cut-off characteristics of SOS, the length of P-region $\left(x_{p}\right)$ is changed from $100 \mu \mathrm{m}$ to $200 \mu \mathrm{m}$ (see Fig. 1). Physical model used here, which considers all relevant equations to simulate the interruption process at temperature $T=300 \mathrm{~K}$, is based on [1]. The space charge along the device for different lengths of P-region and times has been shown in Fig. 1. The interruption process begins when the plasma distribution reaches its equilibrium level making a space charge region due to uncompensated charge carriers. As it can be seen, in all

\footnotetext{
* corresponding author; e-mail: kniayesh@ut.ac.ir
}

cases the current interruption begins from highly doped N-region and ends at highly doped P-region. With the growth of $x_{p}$ the extra plasma and consequently the current have been increased and cut-off time has been decreased (see Fig. 2). It can be seen that with the increase of $x_{p}$ the space charge is formed faster and with higher extent in two highly doped regions causing higher built-in electric field and faster current interruption.

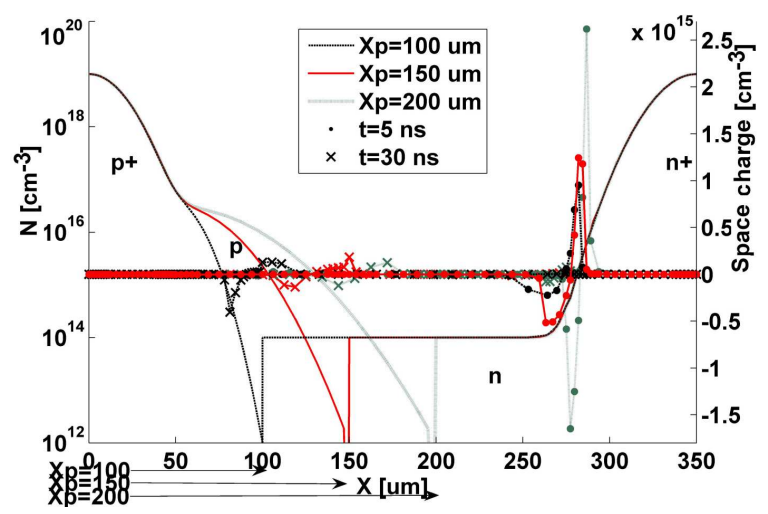

Fig. 1. Doping profile of SOS and space charge formation for three lengths of P-region $\left(x_{p}\right)$ and two time instants. The beginning of current interruption is considered to be $t=0$.

Although the cut-off characteristics (interruption time and interrupted current) can be improved with the increase of $x_{p}$, the hold-off voltage characteristics of the switch is degraded. So, while varying $x_{p}$ one has to make a compromise between hold-off voltage and interruption characteristics. 


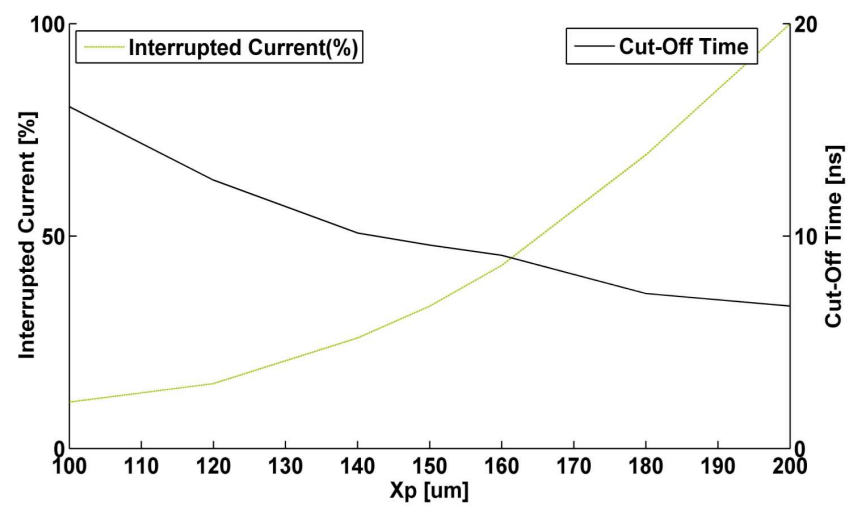

Fig. 2. The variation of cut-off characteristics of SOS with the increase of diffusion length $\left(x_{p}\right)$. The interrupted currents have been normalized with respect to the interrupted current in $x_{p}=200 \mu \mathrm{m}$.

\section{Temperature increase in SOS}

In high pulsed power applications with high repetition rate, the performance of the SOS is affected by the temperature increase in the device. In previous simulations, thermal effects have been neglected given that the SOS is dealing with a single pulse only [2]. To investigate the thermal and electrical behavior of the switch, a detailed physical model has been used which takes into account the effect of non-uniform temperature distribution on the electrical behavior of the device, as well as the temperature dependence of the recombination, electron/hole lifetimes, avalanche, mobility and intrinsic carrier concentration $[3,4]$. The electrodes of the device are assumed as constant temperature boundaries. To numerically accelerate the impact of temperature rise on the device performance and reduce simulation time, a $10 \mathrm{MHz}$ repetition rate has been selected in these simulations.

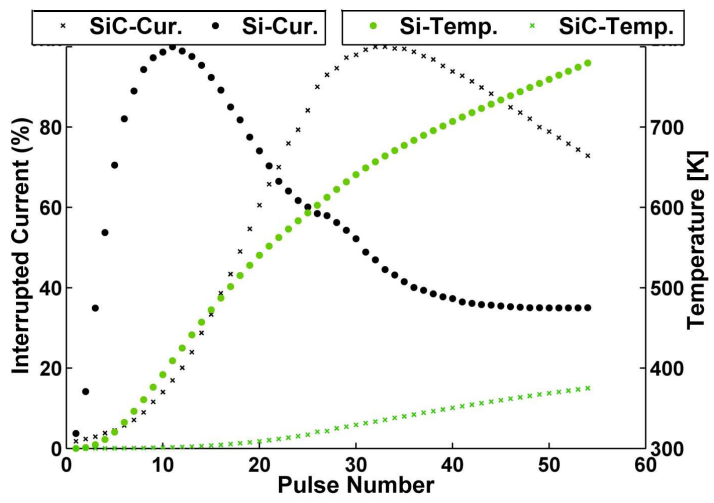

Fig. 3. The variation of interrupted current and temperature with the increase of pulse number. The current curves have been normalized with respect to their own maximum value.

The simulation results for Si-based SOS with $x_{p}=$ $150 \mu \mathrm{m}$ (see Fig. 3), indicate that with the increase of lattice temperature due to high repetition rate and the rise of pulse number, initially the amplitude of the interrupted current is increased and after a decrease, it keeps the level. This contradictory phenomenon can be described considering the initial rise of intrinsic carrier concentration and the decrease in mobility and impact ionization coefficient with temperature. Depending on the technological structure, one or the other effect is predominant [5].

\section{SiC-based SOS}

Silicon carbide $(\mathrm{SiC})$ is a very promising material for use in high power semiconductor devices. Many of the favourable transport parameters in $\mathrm{SiC}$ are related to the large band-gap, which is of the order of $3 \mathrm{eV}$ and due to this large band gap the intrinsic carrier concentration is low $[6]$.

To study the possible application of $\mathrm{SiC}$ as the base material of SOS the same simulation has been performed as the previous section and the result is shown in Fig. 3. As it can be observed, like Si-based SOS the amplitude of the current interrupted has varied with the temperature rise in the device but for the same pulse number, in the $\mathrm{SiC}$-based device the variation of the current amplitude and temperature with increase of pulse number is less than that of the Si-based SOS. The reason is that the thermal conductivity of $\mathrm{SiC}$ is more than three times higher than that of $\mathrm{Si}$, which allows better heat dissipation and consequently the current and temperature sensitivity to the rise of pulse number is decreased. So one can expect that the deviation of the output voltage from nominal value is less in SOS based on $\mathrm{SiC}$ than Si-based device and the cooling system used becomes simpler.

Further improvement of the device performance can be made by increasing the doping level in SiC-based switch. As critical electric field in $\mathrm{SiC}$ is about ten times higher than that of $\mathrm{Si}$, for a given hold-off voltage, doping level $\left(N_{d}\right)$ in SiC-based device can be highly increased which according to $j_{r}=q N_{d} V_{s}$ ( $j_{r}$ and $V_{s}$ are current density and free carriers saturation velocity respectively) leads to higher interrupted current. Thus, favourable characteristics of $\mathrm{SiC}$, i.e. high thermal conductivity, high critical electric field and high current density, result in compact design of high pulsed power high repetition rate devices.

\section{Conclusions}

It was shown that with the increase of $x_{p}$ the cut-off characteristics of SOS are improved, at the expense of decrease in hold-off voltage. Also it was concluded that temperature rise in Si-based SOS leads to high variation of interrupted current and output voltage. To decrease these variations, a SiC-based SOS was studied. It was stated that the mentioned characteristics of $\mathrm{SiC}$ can lead to better performance of device and the design of more compact applications will be possible. 


\section{Acknowledgments}

The authors would like to acknowledge the fruitful discussions with Dr. M. Fathipour.

\section{References}

[1] T.U. Jing, L.U.O. Jinsheng, Y. Rong, Jpn. J. Appl. Phys. 46, 897 (2007).

[2] A. Engelko, H. Bluhm, J. Appl. Phys. 95, 5828 (2004).
[3] M.S. Tyagi, Solid-State Electronics 26, 577 (1983).

[4] Lombardi, S. Manzini, A. Saporito, M. Vanzi, IEEE Transaction on $C A D \mathbf{7 ( 1 1 ) ,} 1164$ (1998).

[5] H. Garrab, B. Allard, H. Morel, S. Ghedira, K. Besbes, International Journal of Numerical Modeling 17, 539 (2004).

[6] K. Bertilsson, Ph.D. Thesis, KTH Microelectronics and Information Technology, 2004. 